\title{
Image Enhancement by Frequency Analysis
}

\author{
Chen $\mathrm{Yao}^{1,2}$, Yan $\mathrm{Xia}^{1}$ and Jiamin Zhu ${ }^{1}$ \\ ${ }^{1}$ The Third Research Institute of Ministry of Public Security, Shanghai, China \\ 2 Shanghai Key Laboratory of Digital Media Processing and Transmission
}

\begin{abstract}
Because of lighting or the quality of CMOS/CCD, poor images are often gained, which greatly affect subjective observation. Image enhancement can improve the contrast of poor image. In our paper, we propose a new image enhancement algorithm based on frequency analysis. A central energy of FFT is utilized for computation of image enhancement factors. A linear mapping is used for image mapping. Finally, some experimental results are shown for illustration of our algorithm advantage.
\end{abstract}

\section{Introduction}

With the development of image processing, a lot of image application depends on image quality. However, distorted images or poor images occurred because of poor $\mathrm{CCD} / \mathrm{CMOS}$ or poor lighting environment. In this case, image enhancement can be used for improve the image quality. Traditional image enhancement normally makes use of histogram equalization. However, this kind of image enhancement method results in colour nonuniformity and illumination inconsistency. Then, gradient histogram's method is applied for image denoising, image deblurring, image restoration etc. [1-11] However, histogram-based is hard to produce rational effects.

In this paper, we propose an image enhancement based on frequency analysis. We find that the central energy of image frequency domain has high relation with image quality. And, we can use a simple mapping function to get enhanced image with central energy computation.

In the rest part of this paper, frequency analysis scheme will be introduced in section 2. Experimental results are provided in section 3 . And finally section 4 concludes the paper.

\section{Proposed algorithm}

In this section, the proposed algorithm will be introduced. Firstly, a non-local mean filter [2] is utilized for image pre-processing. An simple computation of non-local mean is shown as following

$$
N L(I)=\sum W(i, j) \cdot I(i, j)
$$

Where, $\mathrm{W}$ is a weighting coefficients of pixel intensity similarity. Gaussian kernel is adopted for computation of weighted Euclidean distance. After image filtering, we need to transform the image from spatial domain to frequency domain. Fast FFT is used to realize the transformation, which can be descripted as following,

$$
F F T(I)=\sum_{n=0}^{N-1} I_{n} \cdot e^{-i 2 \pi k n / N}
$$

Where, $\mathrm{N}$ are sample points, which is the power of 2 . $\mathrm{k}$ is the image pixel index. In order to get central frequency magnitude, vertical and horizontal transformation is used for FFT transformed image. Then, we get a map of central frequency magnitude. In this magnitude map, we can find that images with different quality show different central energy. Finally, in order to represent the central energy, we use a central entropy to formulate, which is descripted as following,

$$
\operatorname{EN}(F)=\sum_{i=0}^{255} F_{i}^{\nabla} \cdot \log \left(F_{i}^{\nabla}\right)
$$

Where, $\mathrm{F}$ is the frequency transformed image. Then a linear mapping should be used for final enhanced image. Here, we use a global linear approximation method to remap original image into enhanced image.

\section{Experiments}

In this section, different image enhanced results are demonstrated. Our test database utilized surveillance images.

Figure 1 shows an image enhanced example using our algorithm. Figure1(a) and Figure 1(b) are original low quality images. And, Figure 1(c) and Figure 1(d) are the image enhanced results. It can be seen that our method performs a good image enhancement with better contrast, which closes to real surveillance scene. Figure 2 is another example, which also illustrate our advantage. In our last example, entrance and underground parking are 
shown as following. From all of the experimental results, our algorithm shows better processing effect comparing with original image.

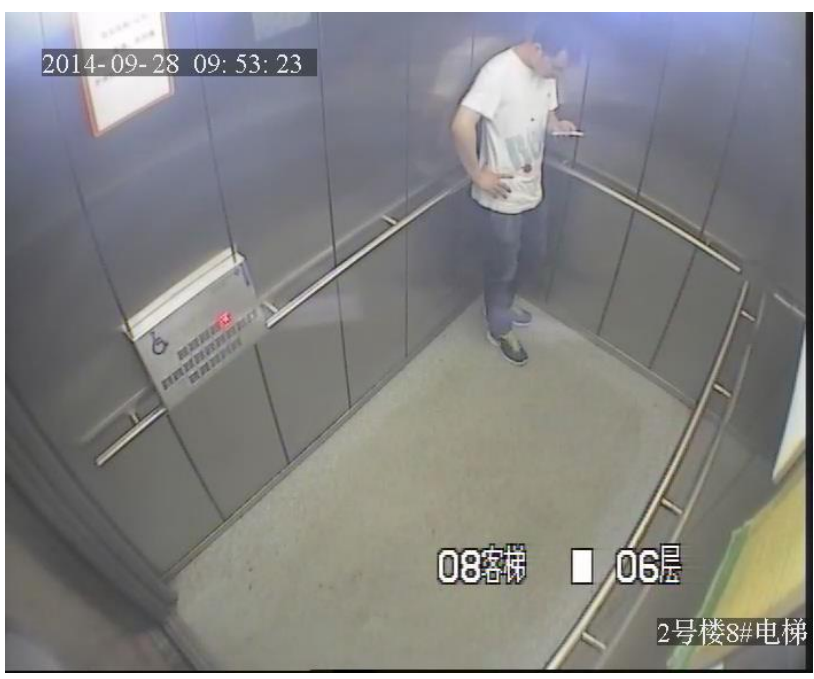

(a)

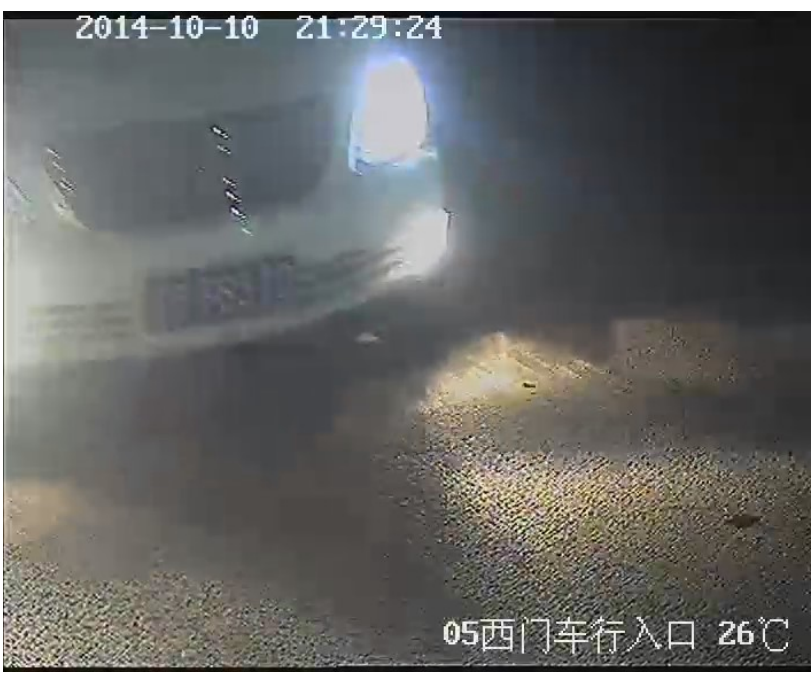

(b)

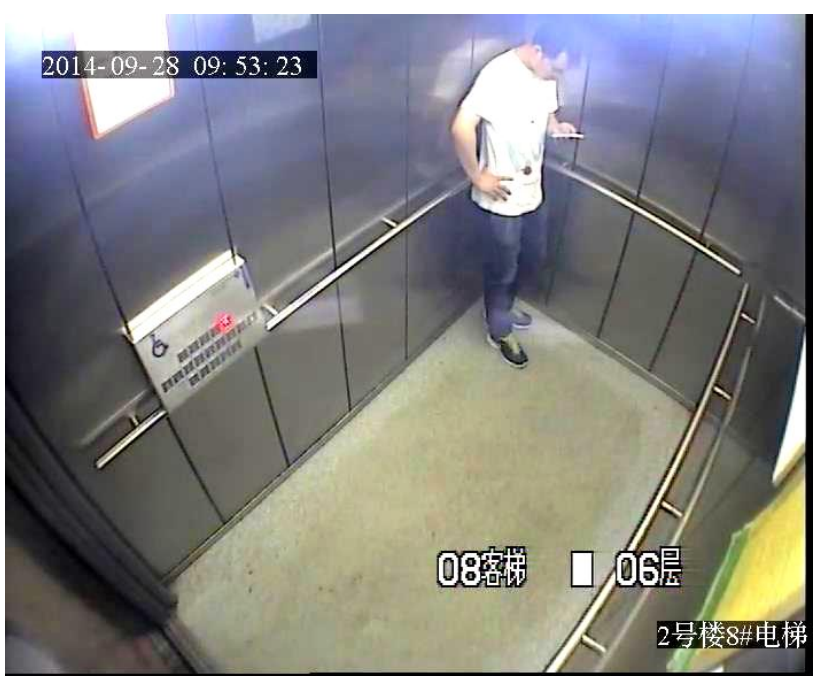

(c)

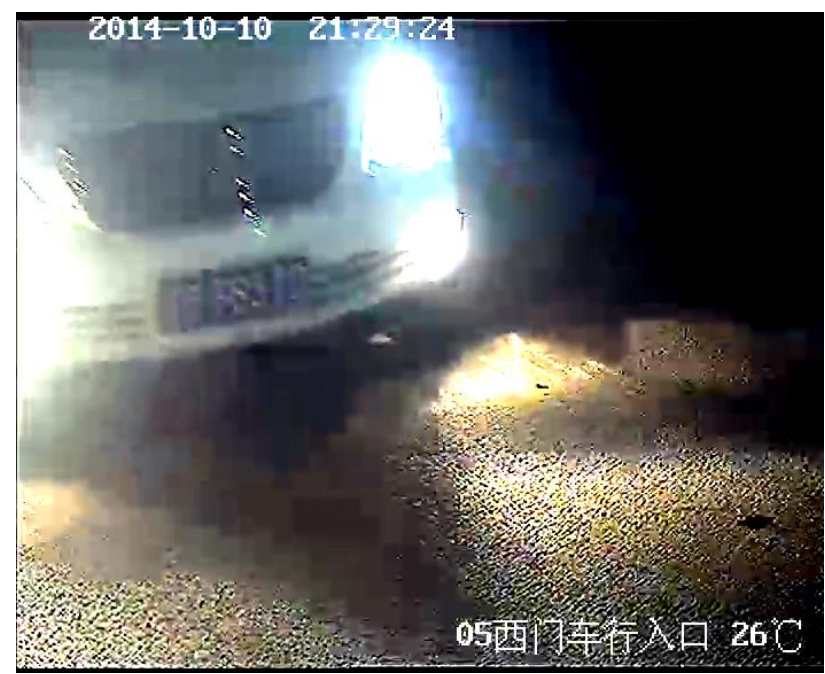

(d)

Figure 1. The effect of image enhancement for surveillance images. $\mathrm{a}$ and $\mathrm{b}$ are input images, $\mathrm{c}$ and $\mathrm{d}$ are enhanced results.

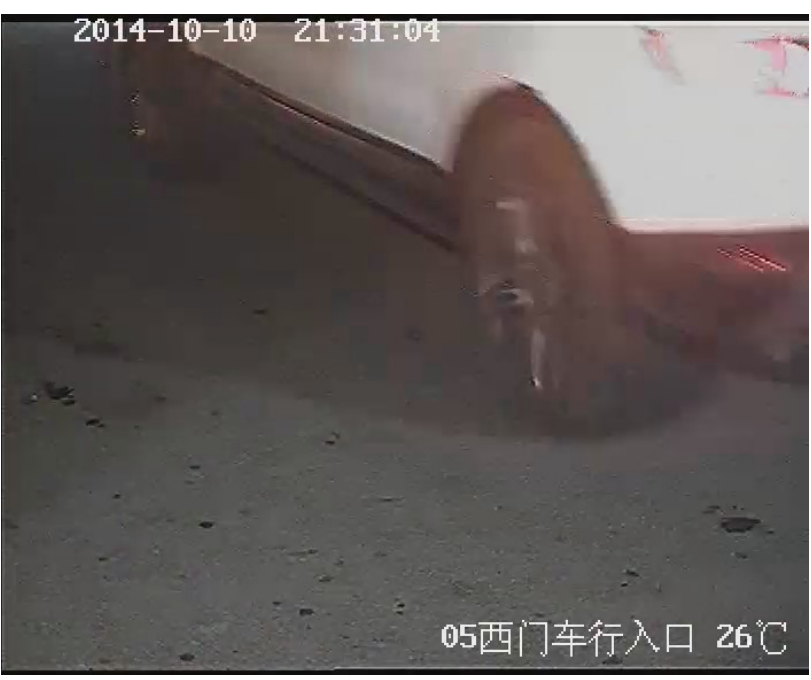

(a)

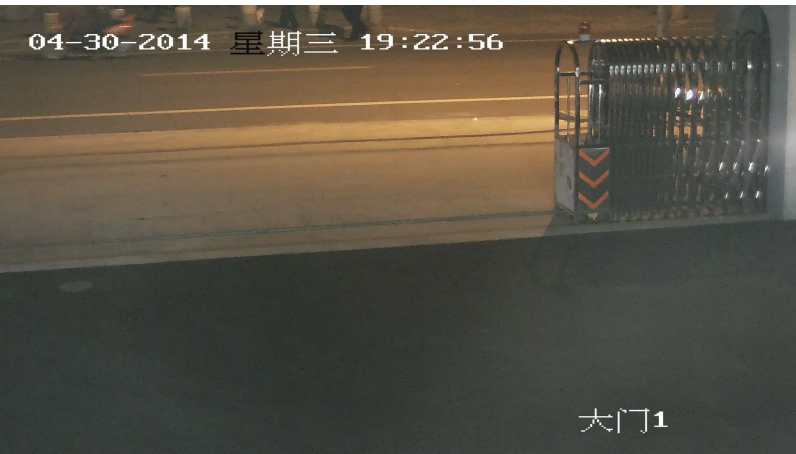

(b) 


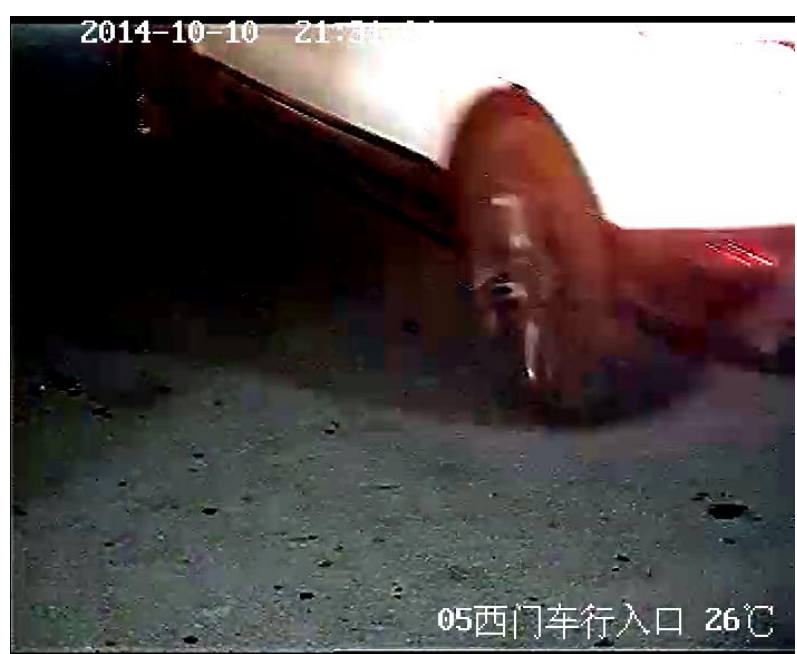

(c)

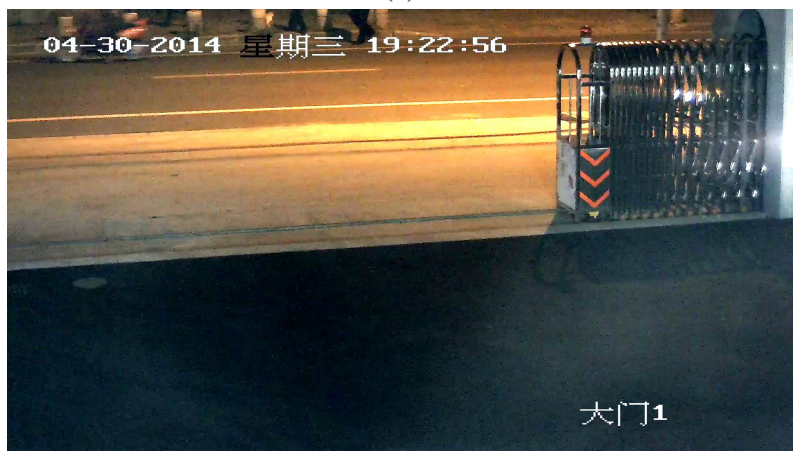

(d)

Figure 2. The effect of image enhancement for surveillance images. $\mathrm{a}$ and $\mathrm{b}$ are input images, $\mathrm{c}$ and $\mathrm{d}$ are enhanced results.

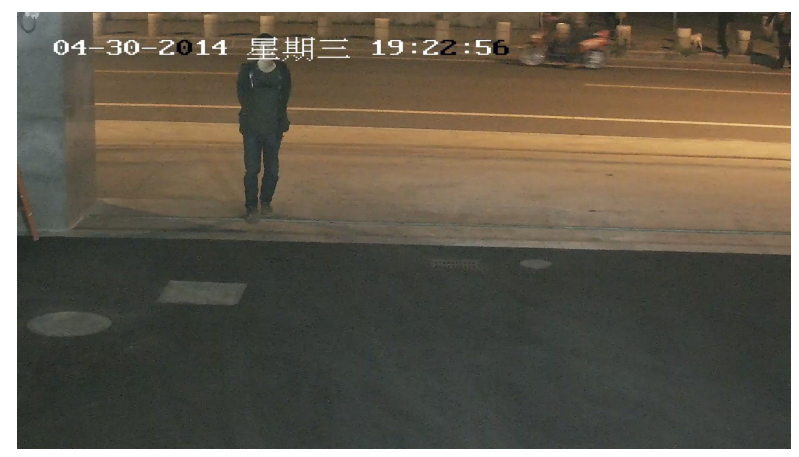

(a)

04-30-2014 星期三 20:15:30

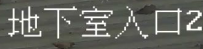

(b)

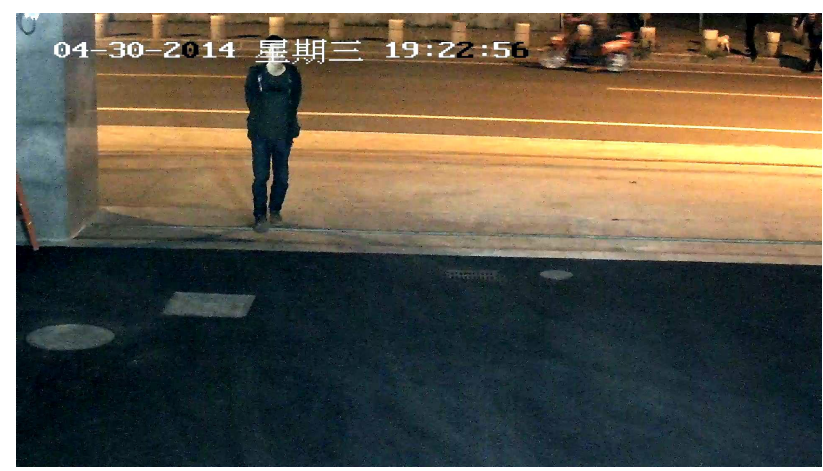

(c)

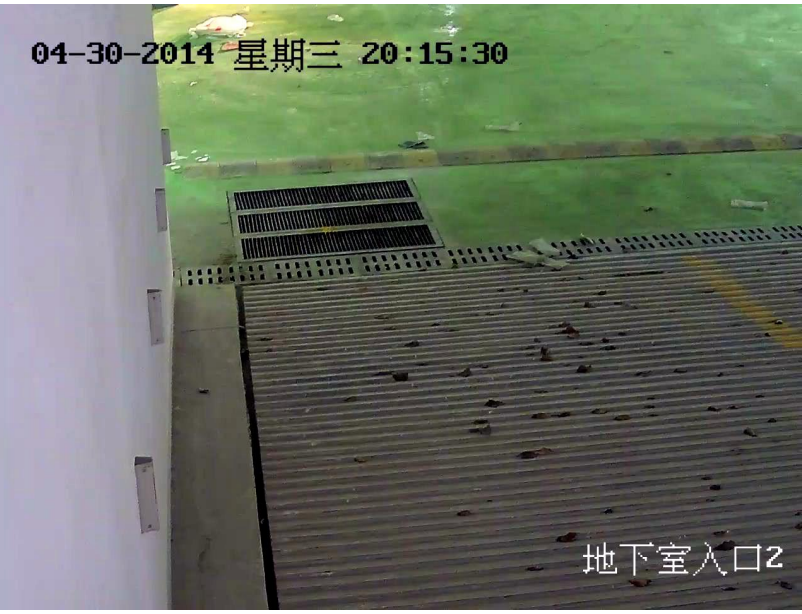

(d)

Figure 3. The effect of image enhancement for surveillance images. $\mathrm{a}$ and $\mathrm{b}$ are input images, $\mathrm{c}$ and $\mathrm{d}$ are enhanced results.

\section{Conclusions}

In this paper, we presented a frequency domain analysis method inspired by frequency central energy fused a linear mapping. Experiment results demonstrate the benefits of frequency domain analysis advantage.

\section{Acknowledgement}

This work was supported by the Opening Project of Shanghai Key Laboratory of Digital Media Processing and Transmission.

\section{References}

1. Chen Y, Yu W, Pock T. On learning optimized reaction diffusion processes for effective image restoration $[\mathrm{C}]$. Proceedings of the IEEE conference on computer vision and pattern recognition. 2015: 5261-5269.

2. Buades A, Coll B, Morel J M. A non-local algorithm for image denoising [C]. Computer Vision and Pattern Recognition, 2005. CVPR 2005. IEEE Computer Society Conference on. IEEE, 2005, 2: 6065.

3. Zhang J, Zhao D, Gao W. Group-based sparse representation for image restoration $[\mathrm{J}]$. IEEE 
Transactions on Image Processing, 2014, 23 (8): 3336-3351.

4. Bar L, Chan T F, Chung G, et al. Mumford and Shah model and its applications to image segmentation and image restoration [J]. Handbook of Mathematical Methods in Imaging, 2014: 1-52.

5. Mao X, Shen C, Yang Y B. Image restoration using very deep convolutional encoder-decoder networks with symmetric skip connections [C]. Advances in neural information processing systems. 2016: 28022810.

6. Katsaggelos A K. Digital image restoration [M]. Springer Publishing Company, Incorporated, 2012.

7. Beck A, Teboulle M. Fast gradient-based algorithms for constrained total variation image denoising and deblurring problems $[\mathrm{J}]$. IEEE Transactions on Image Processing, 2009, 18 (11): 2419-2434.
8. Buades A, Coll B, Morel J M. A review of image denoising algorithms, with a new one [J]. Multiscale Modeling \& Simulation, 2005, 4 (2): 490-530.

9. Starck J L, Candès E J, Donoho D L. The curvelet transform for image denoising [J]. IEEE Transactions on image processing, 2002, 11 (6): 670684.

10. Elad M, Aharon M. Image denoising via sparse and redundant representations over learned dictionaries [J]. IEEE Transactions on Image processing, 2006, 15 (12): 3736-3745.

11. Buades A, Coll B, Morel J M. A non-local algorithm for image denoising $[\mathrm{C}]$. Computer Vision and Pattern Recognition, 2005. CVPR 2005. IEEE Computer Society Conference on. IEEE, 2005, 2: 6065 . 